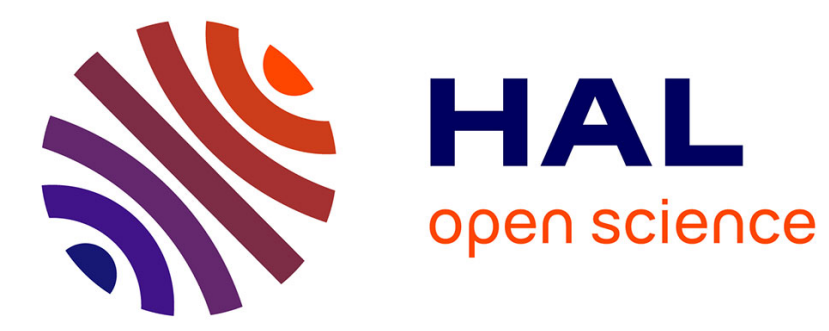

\title{
Attentional sampling of multiple wagon wheels
}

James S P Macdonald, Patrick Cavanagh, Rufin Vanrullen

\section{To cite this version:}

James S P Macdonald, Patrick Cavanagh, Rufin Vanrullen. Attentional sampling of multiple wagon wheels. Attention, Perception, and Psychophysics, 2014, 76, pp.64-72. 10.3758/s13414-013-0555-5 . hal-01298962

\section{HAL Id: hal-01298962 \\ https://hal.science/hal-01298962}

Submitted on 6 Apr 2016

HAL is a multi-disciplinary open access archive for the deposit and dissemination of scientific research documents, whether they are published or not. The documents may come from teaching and research institutions in France or abroad, or from public or private research centers.
L'archive ouverte pluridisciplinaire HAL, est destinée au dépôt et à la diffusion de documents scientifiques de niveau recherche, publiés ou non, émanant des établissements d'enseignement et de recherche français ou étrangers, des laboratoires publics ou privés. 
Running head: Multiple wagon wheels

\title{
Attentional sampling of multiple wagon wheels
}

\author{
James S P Macdonald ${ }^{1}$, Patrick Cavanagh ${ }^{2}$ and Rufin VanRullen ${ }^{3,4}$
}

1. Department of Experimental Psychology, University of Oxford, UK

2. Laboratoire Psychologie de la Perception, Paris Descartes University, CNRS, Paris, France

3. Université de Toulouse, Centre de Recherche Cerveau et Cognition, Université Paul Sabatier, 31052 Toulouse, France

4. CNRS, UMR 5549, Faculté de Médecine de Purpan, 31052 Toulouse, France

Attending to a periodic motion stimulus can induce illusory reversals of the direction of motion. This continuous wagon-wheel illusion (c-WWI) has been taken to reflect discrete sampling of motion information by visual attention. An alternative view is that it is caused by adaptation. Here, we attempt to discriminate between these two interpretations by asking participants to attend to multiple periodic motion stimuli: the discrete attentional sampling account, but not the adaptation account, predicts a decrease of c-WWI temporal-frequency tuning with set size (with a single periodic motion stimulus the c-WWI is tuned to a temporal frequency of $10 \mathrm{~Hz}$ ). We presented one to four rotating gratings that occasionally reversed direction while participants counted reversals. We considered reversal overestimations as manifestations of the c-WWI, and determined the temporal-frequency tuning of the illusion for each set size. Optimal temporal frequency decreased with increasing set size. This outcome favours the discrete attentional sampling interpretation of the c-WWI, with a sampling rate for each individual stimulus dependent on the number of stimuli attended.

Address correspondence to:

Rufin VanRullen, email: rufin.vanrullen@cerco.ups-tlse.fr 


\section{INTRODUCTION}

The 'Wagon Wheel Illusion' is the appearance in movies of a wheel rotating in the opposite direction to its original motion. Such aliasing occurs when the wheel rotates at a slightly slower temporal frequency than the sampling rate of the camera recording it, since with each frame the wheel's spokes appear to have rotated backwards a small amount rather than forwards by a large amount. Surprisingly, a similar illusion can occur when viewing periodic, moving objects in the real world under continuous lighting conditions, such as sunlight (Kline, Holcombe, \& Eagleman, 2004; Purves, Paydarfar, \& Andrews, 1996; Schouten, 1967; Simpson, Shahani, \& Manahilov, 2005; VanRullen, Reddy, \& Koch, 2005), leading many to suggest that motion perception may involve discrete sampling of visual information like a movie camera (Andrews \& Purves, 2005; Crick \& Koch, 2003; Koch, 2004; McComas \& Cupido, 1999; Purves et al., 1996; Rojas, Carmona-Fontaine, López-Calderón, \& Aboitiz, 2006; Simpson et al., 2005; VanRullen \& Koch, 2003; VanRullen et al., 2005). Specifically, it has been proposed that such discrete sampling originates in the attentional system (Reddy, Rémy, Vayssière, \& VanRullen, 2011; VanRullen, Pascual-Leone, \& Battelli, 2008; VanRullen et al., 2005; VanRullen, Reddy, \& Koch, 2006; VanRullen, 2006, 2007), since this ‘continuous’ Wagon-Wheel Illusion (c-WWI) is strongly associated with attention: it occurs far less frequently when attention is drawn away from the periodic stimulus by a concurrent demanding discrimination task (VanRullen et al., 2005), it does not occur in the entire visual field (Kline et al., 2004), but rather in one object at a time that may be formed from several elements according to gestalt principles of association (VanRullen, 2006), and it has been associated with right parietal lobe processing with a variety of neurophysiological techniques (EEG: VanRullen et al., 2006; r-TMS: VanRullen et al., 2008; fMRI: Reddy et al., 2011). According to this proposal, the c-WWI is limited to attended stimuli because discrete sampling in the attentional system leads to aliasing in the attention-based motion perception system, but not in the lower-level motion perception system. Note that previous authors have proposed a distinction between either two (Cavanagh, 1992) or three (Lu 
\& Sperling, 1995) separate motion systems: both theories concurred on the distinction between a lower-level, pre-attentive stage and a higher-level, attention-based system, but differed on the possible existence of an intermediate stage.

An alternative explanation of the c-WWI is that it is a form of motion aftereffect that occurs while the stimulus is still present: a 'motion during-effect' caused by the spurious activation of low-level motion detectors after a period of adaptation (Holcombe, Clifford, Eagleman, \& Pakarian, 2005; Holcombe \& Seizova-Cajic, 2008; Kline \& Eagleman, 2008; Kline et al., 2004; Kline, Holcombe, \& Eagleman, 2006). With this adaptation view of the cWWI, its association with attention is explained by proposing that attention regulates the amount of motion adaptation. Some other features of the c-WWI are also in line with such a proposal: it is a bistable percept that occurs sporadically, and it requires a period of viewing time before it is experienced (Andrews \& Purves, 2005; Holcombe et al., 2005; Kline et al., 2004, 2006; VanRullen, 2007). Other features still, however, are contradictory to this adaptation view, but are compatible with the proposal that it is a consequence of aliasing in at least one motion perception system due to discrete sampling: it has an optimal stimulus temporal frequency (of about $10 \mathrm{~Hz}$ ) (VanRullen et al., 2005), it does not depend on the stimulus' spatial frequency (Purves et al., 1996; Simpson et al., 2005; VanRullen et al., 2005), it occurs for motion of both first-order stimuli (luminance-defined) and second-order stimuli (contrast-defined) (VanRullen et al., 2005), even though these two types of stimuli may be processed by different areas of the brain (Dumoulin, Baker, Hess, \& Evans, 2003; Vaina \& Soloviev, 2004, although see Nishida, Sasaki, Murakami, Watanabe, \& Tootell, 2003; Seiffert, Somers, Dale, \& Tootell, 2003), and its strength decreases with eccentricity, whereas both the static and flicker motion aftereffects increase with eccentricity (VanRullen, 2007), indicating that it is not solely driven by adaptation. Furthermore, the bistability of the c-WWI can be reconciled with the notion of discrete attentional sampling by proposing that adaptation leads to a competition for dominance between the veridical percept generated by the lower-level motion perception system, which is 
unaffected by discrete sampling, and its alias from the attention-based system where the c-WWI originates. The role of adaptation in this proposal is therefore in varying the relative strengths of the veridical percept and its alias over time.

One way in which the discrete attentional sampling and adaptation explanations of the cWWI might be distinguished is by investigating the effect of dividing attention between multiple periodic motion stimuli. The discrete attentional sampling hypothesis predicts that the optimal stimulus temporal frequency for observing illusory reversals of the direction of motion would decrease as the number of attended stimuli increased. This is because each individual stimulus would be sampled in turn, reducing the rate at which any one stimulus is sampled (unless sampling is assumed to be purely parallel and resource-unlimited, a possibility that we address below). The adaptation hypothesis, on the other hand, has no reason to predict a change in optimal stimulus temporal frequency. Instead, it could predict a decrease in illusion strength when attention is divided among multiple stimuli, since adaptation is known to depend on attention (Chaudhuri, 1990; Rees, Frith, \& Lavie, 1997), and the overall amount of attention allocated to each individual target likely decreases with set size.

The expected outcome of the set size manipulation we propose depends on the purported mechanism of divided attention. It is still debated whether attention divides in a sustained fashion to subsume multiple objects (a parallel model), or whether it shifts sequentially between the different simultaneously presented targets (a serial model). A review of the vast and ongoing literature concerning the mechanism of divided attention is beyond the scope of this article, but for a recent review see Jans, Peters, and De Weerd (2010). For our purposes, a serial model would imply that each target is sampled in only a subset of the attentional 'snapshots', as the focus of attention shifts continually between targets. A snapshot of each target would be stored in memory and compared with subsequent snapshots, in order to extract the motion signal for each target individually. This reduction in the sampling rate of individual targets would result in a corresponding reduction in the optimal temporal frequency at which the c-WWI occurs. 
Likewise, a parallel model in which all targets are sampled simultaneously, but with a sampling rate dependent on the complexity of each sample, could make a similar prediction: a decrease of optimal c-WWI temporal frequency with increasing set size. Only a strict parallel model, in which all targets are sampled simultaneously and at the same rate regardless of set size, would predict a constant c-WWI optimal temporal frequency. Since this is the same prediction as the adaptation interpretation, such an outcome would not allow us to differentiate the competing theories.

\section{The relation of c-WWI optimal frequency to attentional sampling rate}

In order to interpret the results of our set-size manipulation, it is important to understand the mathematical relationship between the frequency tuning of the c-WWI and the rate of discrete attentional sampling that we propose causes it. An earlier paper (VanRullen et al., 2005) describes why a maximal c-WWI at $10 \mathrm{~Hz}$ implies that the underlying sampling rate is approximately $13 \mathrm{~Hz}$. The explanation is as follows: The strongest motion signal from a periodic stimulus occurs when the motion steps are 1/4 cycle. Therefore the maximum reverse motion strength will occur when the stimulus steps 3/4 of a cycle with each sample. For sampling of a $10 \mathrm{~Hz}$ signal to fall at the 3/4 cycle point on each sample, the sampling rate must be $4 / 3$ of 10 $\mathrm{Hz}-13.3 \mathrm{~Hz}$. Currently, we only have an estimate of the sampling rate of attention when a single periodic motion stimulus is attended at a time. Kline et al. (2004) did use two simultaneous wagon wheel stimuli, but did not manipulate temporal frequency or measure the optimal temporal frequency of the illusion. We propose to quantify the temporal-frequency tuning of the c-WWI as a function of the number of attended wagon-wheel stimuli. If attention moves from wheel to wheel to capture samples, then the sampling rate should decrease as the number of stimuli increases.

\section{Our study}

We asked participants to report the number of motion direction 'reversal events' (i.e., brief reversals of the direction of motion) occurring in a set of slightly ambiguous, rotating stimuli 
and we varied both the set size (i.e., the number of stimuli presented simultaneously) and the rotation temporal frequency, from trial to trial. Each individual stimulus was composed of two superimposed gratings rotating in opposite directions, with one at a higher contrast than the other so that the perceived motion was biased in that direction. This slightly ambiguous, periodic stimulus was found to be more often seen as reversing. To encourage participants to report all perceptual reversals, several actual motion direction reversals occurred during each 40-second trial; whenever the number of reported reversals was greater than the actual number, we inferred that the difference was the number of illusory reversal events (i.e., instances of the c-WWI) the participant had seen in that trial. We plotted this measure against temporal frequency for each set size, and fit the data points with a Gaussian curve, in order to assess the temporal-frequency tuning of the c-WWI across set sizes.

\section{METHOD}

\section{$\underline{\text { Participants }}$}

12 participants were recruited at the Université Paul Sabatier in Toulouse and were paid for their participation. Three participants were excluded and replaced because they reported very few illusory reversal events. Another three were excluded and replaced because their mean correlation of reported reversals and real reversals across temporal frequencies was below 0.5 (i.e., they were not accurately reporting the number of real reversal events, and hence not performing the task properly; see Procedure for more details of this exclusion criterion). The age range of those included was 21 to 34 years $(\underline{M}=27.2$ years, $\underline{\mathrm{SD}}=3.7$ years $)$, and there were eight males. For the motion after-effect control experiment, four participants were recruited at the University of Oxford, having an average age of 28.3 years ( $\underline{\mathrm{SD}}=3.8$ years), and including one male. All of the participants had normal or corrected-to-normal vision.

\section{$\underline{\text { Apparatus and Stimuli }}$}


The experiments were created and run with Matlab 2007a (The Mathworks, Inc., 2007) on a Windows PC attached to a 17” monitor at a resolution of 1024 x 768 and a refresh rate of 100 Hz. A viewing distance of $57 \mathrm{~cm}$ was maintained throughout the experiment. In each trial, one to four clockwise rotating circular gratings of $1.4^{\circ}$ radius were presented equally spaced but at a randomly determined location on an imaginary circle of $2.5^{\circ}$ radius that was centred at fixation (nearest contours $2.3^{\circ}, 1.6^{\circ}$, and $0.9^{\circ}$ apart for set sizes two to four, respectively). The circular gratings were contained within a disc of $4.7^{\circ}$ radius that was mid-grey $\left(30.3 \mathrm{~cd} / \mathrm{m}^{2}\right)$, and the background of the display was black. A red circle of $0.1^{\circ}$ radius at the centre of the screen served as a fixation point (see Figure 1 for example displays). Each circular grating was in fact composed of two superimposed gratings moving in opposite directions. The contrast difference between the two was adjusted for each participant pre-experimentally so as to make rotation direction weakly biased. Each circular grating had a sinusoidal luminance modulation of eight cycles per rotation. The grating(s) rotated during each 40-s trial at a constant temporal frequency that was selected at random from one of six possibilities: 2.0, 4.0, 5.9, 7.7, 10.0, and $12.5 \mathrm{~Hz}$. In each trial, two to five real reversals of the direction of motion occurred at randomly selected intervals in a subset or in all of the gratings, lasting between 0.5 and $1.5 \mathrm{~s}$ before reverting to the original direction of motion. The minimum time between these real reversal events was $1.5 \mathrm{~s}$ and none occurred during the first 3 s of a trial. A counterbalanced set of 192 different stimulus displays consisting of six temporal frequencies, four set sizes, and four amounts of real reversal events, was presented in four blocks of 48 trials. Each block contained all temporal frequencies and all set sizes, but only two amounts of real reversal events that were counterbalanced across blocks, such that each block contained either two and four, two and five, three and four, or three and five real reversal events. The order in which these pairs of numbers of real reversal events were presented was counterbalanced across participants.

In the motion after-effect control experiment, the same rotating circular gratings were presented for 20 s per trial, at a set size of either one or four, with no direction reversals. The 
same six temporal frequencies were used. At the end of each trial, the circular gratings stopped rotating, and a large red circle appeared around them to emphasise this event. In half of the blocks, the circular gratings remained motionless after stopping rotating - these blocks measured the 'static' motion after-effect; in the other half of the blocks the circular gratings counterphased (flickered) at the same temporal frequency that they had previously been rotating - these blocks measured the 'flicker' motion after-effect (e.g., Nishida \& Sato, 1995, see Mather, Pavan, Campana, \& Casco, 2008 for a review). A counterbalanced set of 24 different stimulus displays consisting of six temporal frequencies, two set sizes, and two motion directions was presented in each of four blocks. Blocks alternated between measuring the static and flicker motion after-effects.

\section{Procedure}

The contrast difference between each pair of superimposed gratings was adjusted for each participant pre-experimentally so as to be somewhat ambiguous in rotation direction but not indistinguishable. This was achieved by displaying a series of these biased motion stimuli (pairs of superimposed gratings) of increasing contrast difference for 10 seconds each, and asking the participant if the direction of motion was clear and if so, whether it was clockwise or anticlockwise. The first contrast difference at which the participant was able to state the correct direction of motion with confidence was selected to be used for the first block of 48 trials. Next, each participant completed a short practice block consisting of eight randomly-selected trials before starting the experiment proper. These practice trials were identical to the trials in the experimental blocks, which proceeded as follows (see Figure 1): each trial's stimulus display lasted $40 \mathrm{~s}$, after which the rotating gratings stopped moving and above them in white the words, 'How many reversal events did you see? Enter digit', appeared. The participants were instructed to report the number of reversal events they had seen during the trial at this point by pressing one of the keys on the numeric keypad (0-9). If they had seen more than nine reversal events they were instructed to report nine. A reversal event was defined as a brief reversal of the 
direction of motion in one or more stimuli followed by a return to the original direction of motion. Responses were not speeded and no feedback was given. Upon response the next trial began. After each 48-trial block the contrast difference between the two superimposed gratings (and thus, the ambiguity of the motion direction) could be raised or lowered in order to adjust task difficulty, depending on whether the participant had seen very many or very few illusory reversal events across all temporal frequencies.

In order to verify that participants were performing the reversal event counting task and were attending effectively to all the gratings, we calculated the Pearson correlation of the number of reported reversal events with the number of real reversal events for each participant, separately for each of four blocks and six temporal frequencies. If the average correlation across temporal frequencies for any block was below 0.5 the block was excluded from the analysis. With this criterion, three participants were excluded from the analysis, since all of their blocks failed, and were replaced with three new participants; five participants had two blocks excluded, and one participant had one block excluded. The remaining six participants had no blocks excluded. The mean correlation across participants after exclusion of blocks was 0.66 (SD = 0.10). Note that the occurrence of the illusion is bound to affect this correlation value; however, given a particular illusion strength, on average more actual reversals should always result in more reported reversals if participants are performing the counting task correctly. Thus, the presence of a positive correlation is a valid marker of reversal-counting performance.

In the motion after-effect control experiment, participants were instructed to observe the rotating gratings whilst maintaining fixation, and when they had stopped rotating, wait until the motion after-effect (illusory motion in the opposite direction) had stopped before pressing the ' 0 ' key on the numeric keypad. Participants pressed the ' $\mathrm{S}$ ' key to initiate each trial and there was a four-second inter-trial interval. 


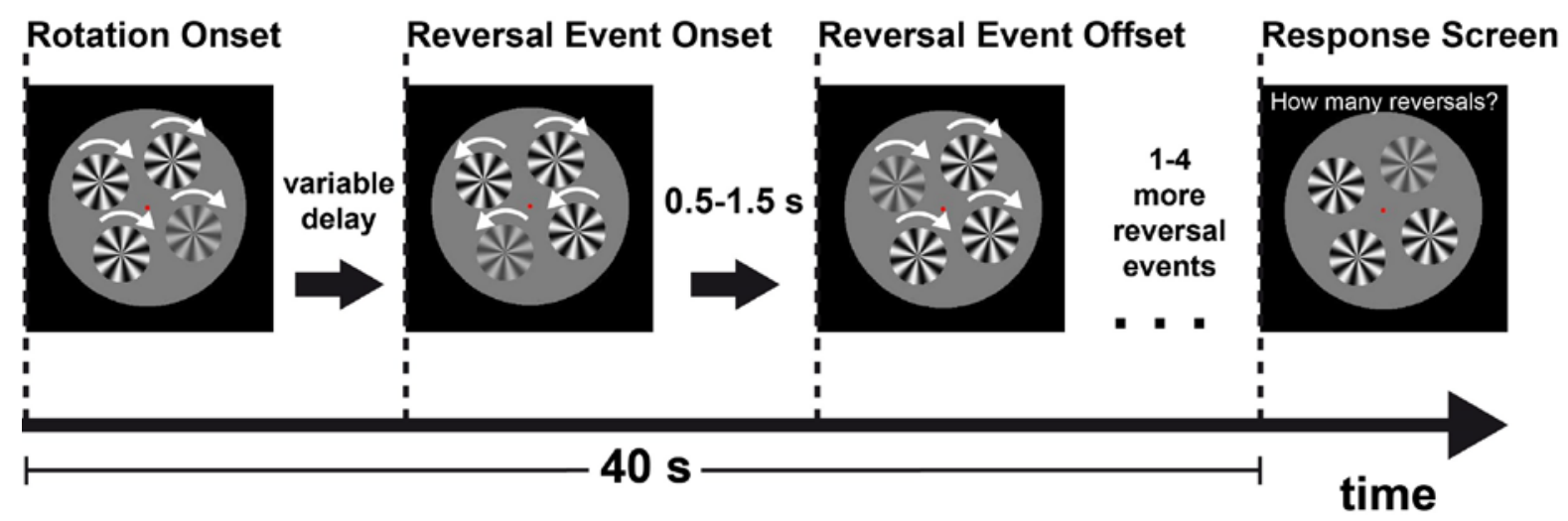

Figure 1. Experimental Procedure. Each trial display contained one to four circular gratings rotating clockwise with some ambiguity in the rotation direction introduced by superimposing two counter-phased gratings of opposite directions and slightly different contrasts. Each trial lasted for $40 \mathrm{~s}$, during which time two to five actual 'reversal events' (lasting between 0.5 and 1.5 s) occurred in a subset or all of the gratings. A 'reversal event' was defined as a brief, single reversal of the direction of motion simultaneously involving any number of gratings, followed by a return to the original direction of motion. There was a delay of at least $1.5 \mathrm{~s}$ between reversal events. Participants were instructed to report the number of reversal events they had observed at the end of each trial.

\section{RESULTS}

\section{Continuous Wagon-Wheel Illusion temporal-frequency tuning}

Our experimental procedure gave us, for each of four blocks per participant, the number of reported reversal events in each trial to compare to the number of actual reversal events. We considered overestimations of the number of reversals as manifestations of the c-WWI, and for each participant we calculated the average ratio of reported reversal events to actual reversal events across trials as a function of set size and temporal frequency. After averaging these ratios across participants, we fitted the temporal-frequency tuning of the illusion for each set size with a Gaussian function (Figure 2) with four parameters: mean $(\mu)$, standard deviation $(\sigma)$, amplitude (A), and baseline (b). The fits minimized the error term $|\xi|$ in the following equation: 
$r r(f)=\frac{A}{\sqrt{2 \pi} \sigma} e^{-(f-\mu)^{2} / 2 \sigma^{2}}+b+\xi(f)$

where $\underline{\mathrm{f}}$ is the temporal frequency of the stimulus and $\underline{\mathrm{rr}}$ is the 'reversal ratio' between reported and actual reversal events. Recall that the attentional sampling interpretation of the cWWI predicts a drop in temporal-frequency tuning with increasing set size, whereas the adaptation interpretation a priori does not.

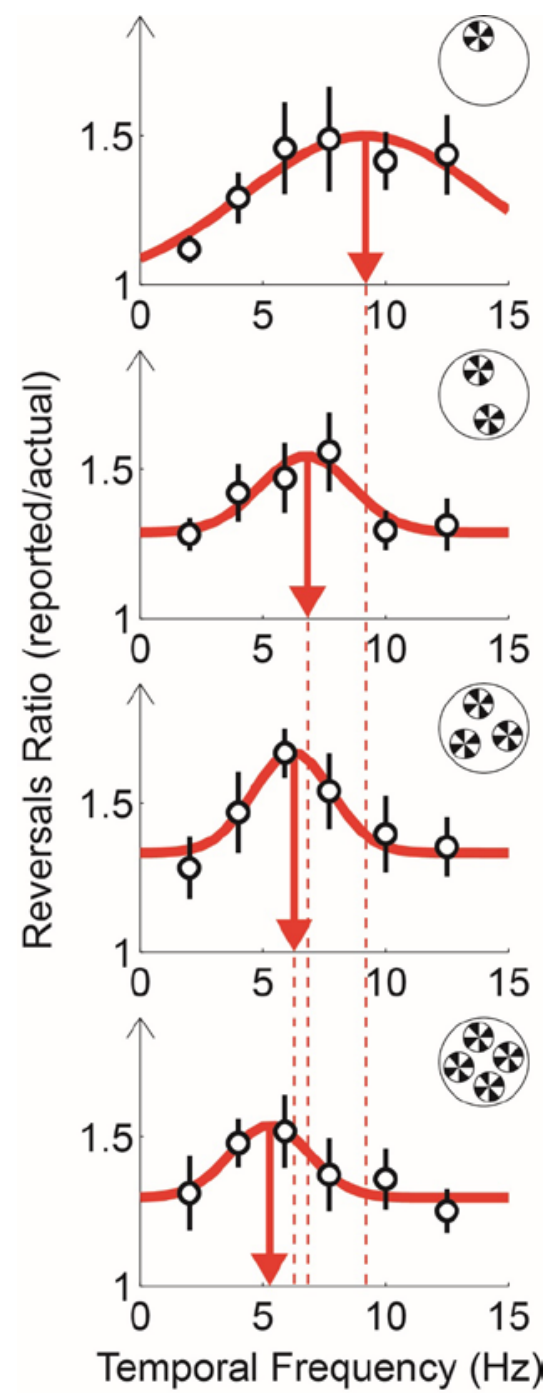

Figure 2. Mean reversal ratio (reported/actual) as a function of circular grating rotation temporal frequency for each set size. Error bars indicate the standard error of the mean. The average ratio of reported reversal events to actual reversal events is taken as a marker of the occurrence of the c-WWI: ratios above one indicate instances of illusory reversals in addition to actual reversals. 
The data points for each set size were fitted with a Gaussian function in order to determine the optimal temporal frequency of the c-WWI (represented by a red vertical arrow in each case). The optimal temporal frequency decreased significantly with increasing set size $(\mathrm{p}<.001)$.

The data points for each set size fit a Gaussian function well, the mean of which represents the stimulus temporal frequency most likely to induce an illusory reversal of the direction of motion (the optimal temporal frequency of the c-WWI). For set size one, the mean of our fitted Gaussian, and hence the optimal temporal frequency, was $9.2 \mathrm{~Hz}$, a figure very close to the optimal temporal frequency found previously for a single periodic rotating stimulus, $10 \mathrm{~Hz}$ (VanRullen et al., 2005). Our data for set size one therefore replicate this previous finding and, importantly, also confirm that our new c-WWI paradigm engages similar perceptual mechanisms to the paradigms in previous work on the c-WWI (VanRullen et al., 2005).

With regard to the effect of set size on optimal temporal frequency, inspection of Figure 2 suggests that optimal temporal frequency decreased consistently with increasing set size, from approximately $10 \mathrm{~Hz}$ for set size one to just over $5 \mathrm{~Hz}$ for set size four. In order to test these data statistically, we fitted the temporal-frequency tuning of the illusion for each set size with a Gaussian function in the same way as before but for each participant individually. The means of these Gaussian fits (i.e., the illusion optimal temporal frequency) were entered into a repeated measures one-way analysis of variance (ANOVA) with set size as a factor that revealed a significant decrease of optimal temporal frequency with increasing set size, $\underline{F}(3,33)=8.65$, $\underline{\text { MSE }}=16.27, \underline{\mathrm{p}}<.001, \eta_{\mathrm{p}}{ }^{2}=.44$ (two tailed, as are all further statistical tests). These data are shown in Table 1 and are plotted in Figure 3(a).

Table 1. Grand mean (and SEM) of Gaussian fit parameters (mean, standard deviation, amplitude, and baseline) performed individually for each participant, and peak reversal ratio, as a function of set size. 


\begin{tabular}{|c|c|c|c|c|c|c|c|c|c|c|}
\hline Set Size & \multicolumn{2}{|c|}{ Mean $(\mu$, in Hz) } & \multicolumn{2}{c|}{$\begin{array}{c}\text { Stand. Dev. } \\
(\sigma, \text { in Hz })\end{array}$} & \multicolumn{2}{|c|}{ Amplitude (A) } & \multicolumn{2}{|c|}{ Baseline (b) } & \multicolumn{2}{|c|}{ Peak Rev. Ratio } \\
\hline & M & SD & M & SD & M & SD & M & SD & M & SD \\
\hline 1 & 8.61 & 0.76 & 3.76 & 0.71 & 9.91 & 3.16 & 0.84 & 0.12 & 1.76 & 0.16 \\
\hline 2 & 6.58 & 0.80 & 3.82 & 0.65 & 7.37 & 2.42 & 0.95 & 0.11 & 1.73 & 0.12 \\
\hline 3 & 6.38 & 0.65 & 2.88 & 0.30 & 7.55 & 2.51 & 0.93 & 0.11 & 1.84 & 0.10 \\
\hline 4 & 6.01 & 0.60 & 2.63 & 0.23 & 4.24 & 1.05 & 1.06 & 0.04 & 1.73 & 0.14 \\
\hline
\end{tabular}

Pairwise comparisons revealed that optimal temporal frequency for set size one was significantly higher than that for set sizes two, three, and four (all $\mathrm{p}<.005$ ). Our data therefore suggest that attending to multiple objects reduces the rate of sampling of each individual object compared to the rate of sampling for a single attended object. This finding is in line with the prediction derived from the discrete attentional sampling interpretation of the c-WWI.

We performed the same ANOVA analysis that we had used on the mean for the other parameters of the Gaussian fits (i.e., standard deviation, amplitude, and baseline - all shown in Table 1). None of these three ANOVA were significant, however, therefore our data do not indicate an effect of set size on temporal-frequency tuning standard deviation, $\underline{F}(3,33)=1.55$, $\underline{\mathrm{MSE}}=4.44, \underline{\mathrm{p}}=.219$, amplitude, $\underline{\mathrm{F}}(3,33)=1.79, \underline{\mathrm{MSE}}=65.15, \underline{\mathrm{p}}=.169$, or baseline, $\underline{\mathrm{F}}(3,33)$ $=1.29, \underline{\mathrm{MSE}}=0.094, \underline{\mathrm{p}}=.295$, none of which we expected to find.

\section{Continuous Wagon-Wheel Illusion strength}

One prediction that could be derived from the adaptation interpretation of the c-WWI is that illusion strength would decrease with increasing set size, since the amount of adaptation depends on the allocation of attention, which in turn depends on the number of simultaneously attended objects. We took the peak reversal ratio derived from the Gaussian fit for each set size as a measure of the strength of the illusion (presented in Figure 3b), and performed a one-way 
ANOVA on peak reversal ratio with set size as the factor. This analysis did not provide evidence that c-WWI strength varied with set size, $\underline{\mathrm{F}}<1$. This result contrasts with the decrease in optimal temporal frequency with increasing set size (presented in Figure 3a).

(a) Optimal frequency

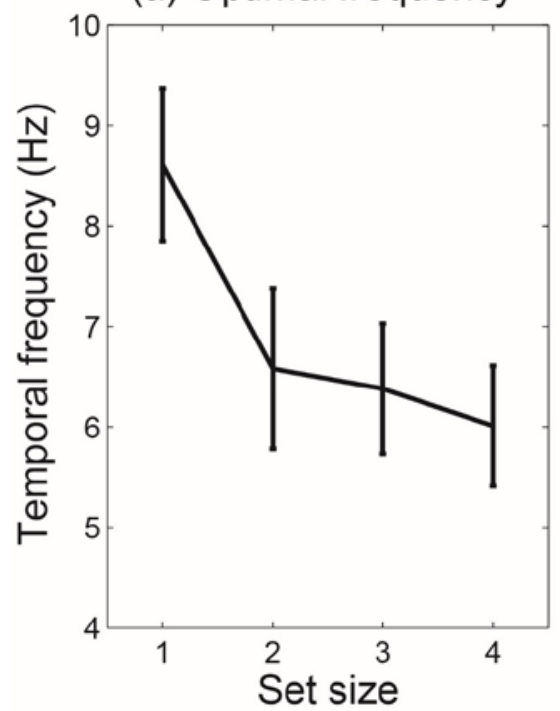

(b) Peak reversal ratio

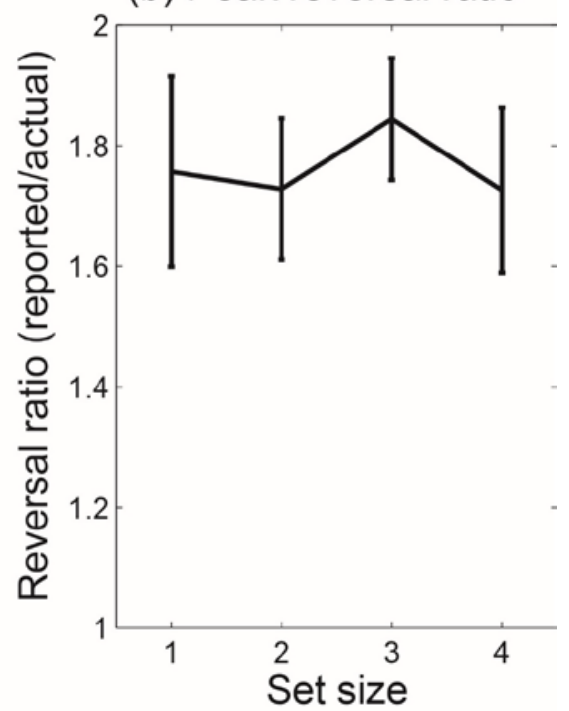

Figure 3. (a) c-WWI optimal temporal frequency (i.e., the grand average of the means of the fitted Gaussians) as a function of set size. (b) c-WWI strength (i.e., the grand average of the peak reversal ratio) as a function of set size. Error bars in both (a) and (b) indicate the standard error of the mean. Optimal temporal frequency decreased with increasing set size, whereas illusion strength was unchanged, supporting the discrete attentional sampling interpretation of the c-WWI over the adaptation interpretation.

\section{Motion after-effect temporal-frequency tuning}

Although the adaptation interpretation of the c-WWI would a priori predict no change in temporal-frequency tuning with set size, one a posteriori explanation could be that adaptation of different populations of neurons, which are differentially tuned to rotation temporal frequency, is responsible for the effect of set size on optimal temporal frequency. For example, observation of a single rotating circular grating might activate motion-sensitive neurons with large receptive 
fields tuned to higher temporal frequencies, whereas observation of multiple rotating circular gratings might instead involve neurons with smaller receptive fields tuned to lower temporal frequencies. To evaluate this interpretation of our results, we carried out a second experiment in which participants viewed either one or four circular gratings rotating at the same six temporal frequencies as in the main experiment and reported the duration of the motion after-effect (MAE) after the gratings had stopped rotating and either remained motionless (static MAE) or counterphased (flicker MAE). As can be observed in Figure 4, the static and flicker MAE duration showed no significant variation with temporal frequency and this absence of tuning was unaffected by set size. This was confirmed by two-way ANOVA on MAE duration with set size and temporal frequency as factors: $\underline{\mathrm{F}}<1$ for the interaction of set size and temporal frequency for both the static and flicker MAE. There was also no main effect of temporal frequency, both $\underline{F}<1$. There was no main effect of set size on the duration of the flicker MAE, $\underline{F}<1$, although there was for the static MAE, $\underline{\mathrm{F}}(1,3)=11.09, \underline{\mathrm{MSE}}=2.02, \underline{\mathrm{p}}=.048, \eta_{\mathrm{p}}{ }^{2}=.79$, with longer MAE durations for the larger set size. This effect could be a result of the larger overall adapted area. Nevertheless, since the temporal-frequency tuning of the MAE was unaffected by set size, this experiment does not support an adaptation interpretation of the relation between the c-WWI optimal temporal frequency and set size. 


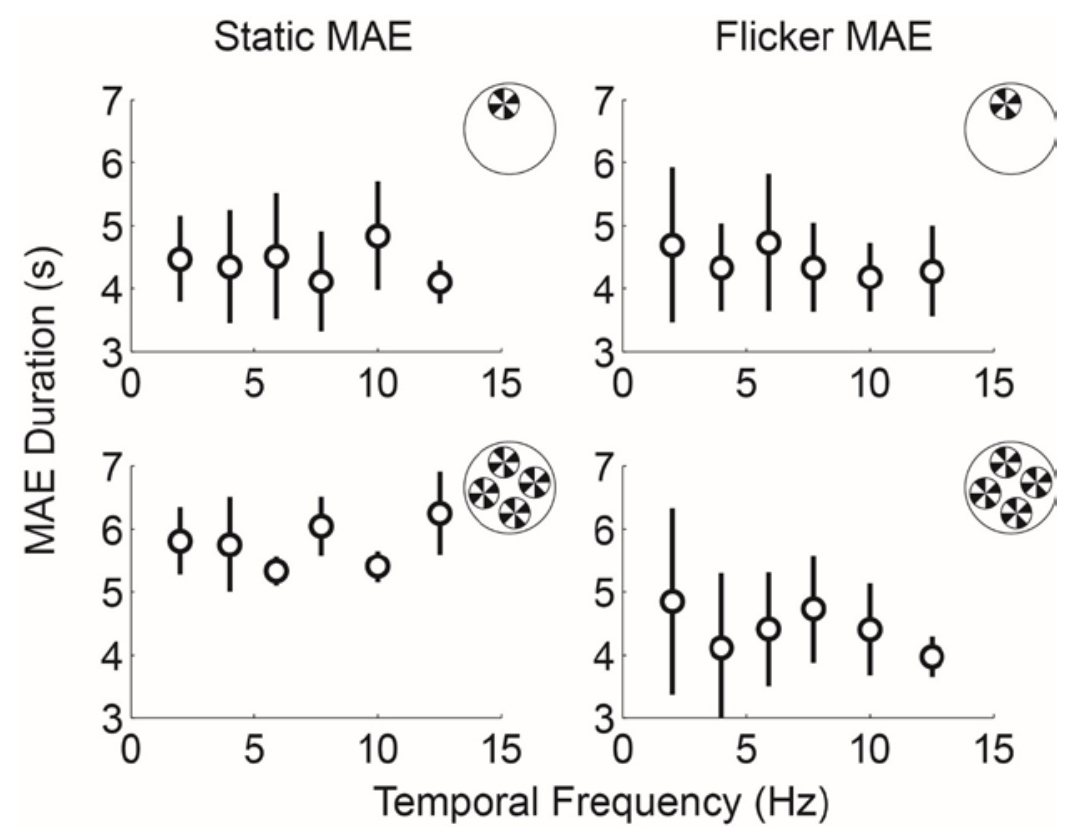

Figure 4. Grand average motion after-effect duration for static (left column) and flicker (right column) test patterns as a function of set size (one on upper row and four on lower row) and circular grating rotation temporal frequency. Error bars indicate the standard error of the mean. Motion after-effect duration temporal-frequency tuning did not vary with set size, ruling out an explanation of the c-WWI optimal temporal frequency set size effect in terms of adaptation of different populations of motion-sensitive neurons in the different set size conditions.

\section{DISCUSSION}

The stimulus temporal frequency most likely to induce an illusory reversal of the direction of motion for set size one was very similar to that found previously for a single periodic rotating stimulus (VanRullen et al., 2005). This figure, $10 \mathrm{~Hz}$, corresponds to an attentional sampling rate of $13.3 \mathrm{~Hz}$ (see Introduction and ibid.). The data for set size one therefore reinforce the claim that attention samples visual information discretely at around $13 \mathrm{~Hz}$ (VanRullen et al., 2005, 2006).

Our manipulation of set size was intended to distinguish between the discrete attentional sampling and adaptation interpretations of the c-WWI, since each makes a distinct prediction about the effect of set size. The discrete attentional sampling interpretation leads to the 
prediction that optimal temporal frequency would reduce as the number of attended stimuli increases, since the sampling rate of each individual stimulus would be a proportion of the sampling rate of a singularly attended stimulus. The adaptation interpretation, on the other hand, gives rise to no a priori rationale for a change in optimal temporal frequency with set size. Instead, a decrease in illusion strength with increasing set size might be predicted, since each of multiple stimuli would receive less attention than a lone attended stimulus, and adaptation is known to scale with attention (Chaudhuri, 1990; Rees et al., 1997). We found that for set sizes two, three and four, in which attention was divided between stimuli, optimal temporal frequency decreased compared to set size one and illusion strength remained unchanged, in line with the discrete attentional sampling interpretation of the c-WWI, and in opposition to the predictions of the adaptation interpretation.

It is difficult to reconcile the finding that optimal temporal frequency decreases with set size with the adaptation interpretation of the c-WWI. According to this account, illusory reversals occur due to the spurious activation of low-level motion detectors after a period of adaptation (Holcombe et al., 2005; Holcombe \& Seizova-Cajic, 2008; Kline \& Eagleman, 2008; Kline et al., 2004, 2006), and the optimal temporal frequency of $10 \mathrm{~Hz}$ of the c-WWI is the consequence of the tuning of low-level motion detectors (Reichardt detectors, Reichardt, 1961) being concentrated around $10 \mathrm{~Hz}$ (Kline et al., 2006), since that is the maximal frequency range of normal human motion sensitivity (Snowden \& Hess, 1992). An account of our results in terms of such motion detectors would therefore have to surmise that an increase in the load on attention, for example, the number of attended objects, results in the spurious activation of a different subset of these detectors that are tuned to temporal frequencies lower than $10 \mathrm{~Hz}$. For example, observation of a single rotating circular grating might activate motion-sensitive neurons with large receptive fields tuned to higher temporal frequencies, whereas observation of multiple rotating circular gratings might instead engage neurons with smaller receptive fields tuned to lower temporal frequencies. We did not find support for such a hypothesis in our 
motion after-effect control experiment, in which the temporal-frequency tuning of MAE duration did not vary with set size. We suggest it is more parsimonious to interpret our results in terms of discrete attentional sampling. Furthermore, since set size effects are considered a hallmark of attentional involvement, our results bolster support for the discrete attentional sampling explanation of the c-WWI in general.

We also considered whether our results could be explained by the occurrence of saccades during the observation of multiple rotating stimuli: if participants had ignored the instruction to maintain their gaze on the fixation point at the centre of the screen, they may instead have shifted their gaze between stimuli at set sizes two and above. Had it occurred, the effect of this intermittent input may have generated additional aliasing artefacts which could have contributed to the downward shift in c-WWI optimal temporal frequency from around $10 \mathrm{~Hz}$ at set size one to 5-7 $\mathrm{Hz}$ at larger set sizes. There are problems with this interpretation, however, that render it unlikely in our opinion. Eye movements can occur at up to 5-6 Hz, or every 160-200 ms (Rayner, 1998). Therefore the simplest hypothesis is that regular shifts of fixation would have generated a secondary peak in illusory reversals at temporal frequencies below 3-4 Hz - about three quarters of eye movement frequency (see the introduction for an explanation of why this would be so). We did not observe any such bimodality in our data. A refined hypothesis could be that several additional peaks were generated, not just at the eye movement switching frequency, but also at harmonic multiples of this frequency. These multiple peaks within the range we presented $(2-12.5 \mathrm{~Hz})$ were also not visible in our data. Finally, the possibility that these multiple peaks could have merged and induced a general baseline increase for set sizes two and above compared to set size one is contradicted by the absence of a significant baseline effect (see Table 1).

We note that in one recent study (Holcombe \& Chen, 2013) the authors reached a similar conclusion to our own - that the sampling rate of attention decreases when the number of attended targets increases - in the context of multiple-object tracking. Holcombe and Chen 
reported that the ability to track objects situated on rotating concentric circles was determined by the temporal frequency of the circles. Critically, as the number of objects to be tracked increased, the maximum temporal frequency at which tracking could be performed reliably decreased. The authors highlighted that only a serial model of attention to multiple objects would a priori predict such an effect, whereas a parallel model would not.

Despite the evidence favoring the discrete attentional sampling proposal, there is one aspect of the results that requires an amendment to the simplest version of sampling: the effect of set size was smaller than would be predicted by a purely serial mechanism of divided attention. Specifically, the optimal temporal frequency of the illusion did not drop linearly with the reciprocal of the number of items sampled (if it had, we would have obtained optimal temporal frequencies of around 5, 3.3, and $2.5 \mathrm{~Hz}$ for set sizes two, three, and four, respectively). On the other hand, our data are also incompatible with a strict parallel and resource-unlimited model, since this model would predict no change in optimal temporal frequency with progressive increases in set size. Therefore, a more complex model of attentional deployment must be invoked to explain our results. We propose three such models: firstly, a modified parallel strategy, in which the sampling rate of attention decreases when the contents of attention are more complex (e.g., when more than one object must be attended); secondly, an irregular serial strategy, in which attentional sampling rate is maintained at approximately 13 $\mathrm{Hz}$, but a variable number of samples are taken from each successively attended object; thirdly, a faster serial strategy, in which individual objects are selected sequentially, but sampling rate increases with set size.

Although we are not able to further disentangle these three models of attentional deployment with the current data, it may be possible to do so in the future by repeating the experiment while recording participants' EEG along the same lines as the study conducted by VanRullen et al. (2006), in which EEG power in the right parietal lobe at $13 \mathrm{~Hz}$ changed with the onset and offset of illusory reversals of the direction of motion: the modified parallel 
strategy would predict that EEG signatures of the c-WWI would occur at lower frequencies as set size increased, the irregular serial strategy would predict no influence of set size on the frequency of the EEG correlate, and the faster serial strategy would predict changes in the EEG power spectrum at successively higher frequencies as set size increased.

The three models we have proposed are necessarily based on simplifying assumptions. For example, we assume a uniform attentional mechanism across space. On the other hand, it is possible that distinct attentional mechanisms exist in the two hemispheres (Alvarez \& Cavanagh, 2005). Our experimental procedure, however, does not allow us to investigate the potential effects of this factor, since perceived motion reversals cannot be unambiguously attributed to one hemisphere or the other.

To summarise, we appraised the predictions of two competing interpretations of the cWWI, namely discrete attentional sampling and adaptation, in a situation where multiple periodic stimuli are attended simultaneously. The optimal temporal frequency of the illusion decreased with increasing set size whereas illusion strength did not, in line with the discrete attentional sampling interpretation of the c-WWI and contrary to the adaptation account.

\section{ACKNOWLEDGEMENTS}

This research was funded by a EURYI Award to Rufin VanRullen.

\section{REFERENCES}

Alvarez, G. A., \& Cavanagh, P. (2005). Independent resources for attentional tracking in the left and right visual hemifields. Psychological Science, 16(8), 637.

Andrews, T. J., \& Purves, D. (2005). The wagon-wheel illusion in continuous light. Trends in Cognitive Sciences, 9(6), 261-263.

Cavanagh, P. (1992). Attention-based motion perception. Science (New York, N.Y.), 257(5076), 1563-1565. 
Chaudhuri, A. (1990). Modulation of the motion aftereffect by selective attention. Nature, 344(6261), 60-62. doi:10.1038/344060a0

Crick, F., \& Koch, C. (2003). A framework for consciousness. Nature Neuroscience, 6(2), 11926. doi:10.1038/nn0203-119

Dumoulin, S. O., Baker, C. L., Jr, Hess, R. F., \& Evans, A. C. (2003). Cortical specialization for processing first- and second-order motion. Cerebral cortex (New York, N.Y.: 1991), 13(12), 1375-1385.

Holcombe, A. O., \& Chen, W.-Y. (2013). Splitting attention reduces temporal resolution from 7 $\mathrm{Hz}$ for tracking one object to $<3 \mathrm{~Hz}$ when tracking three. Journal of vision, 13(1). doi:10.1167/13.1.12

Holcombe, A. O., Clifford, C. W. G., Eagleman, D. M., \& Pakarian, P. (2005). Illusory motion reversal in tune with motion detectors. Trends in Cognitive Sciences, 9(12), 559-60; author reply 560-1. doi:10.1016/j.tics.2005.10.009

Holcombe, A. O., \& Seizova-Cajic, T. (2008). Illusory motion reversals from unambiguous motion with visual, proprioceptive, and tactile stimuli. Vision Research, 48(17), 17431757.

Jans, B., Peters, J. C., \& De Weerd, P. (2010). Visual spatial attention to multiple locations at once: the jury is still out. Psychological Review, 117(2), 637-684. doi:10.1037/a0019082

Kline, K. A., \& Eagleman, D. M. (2008). Evidence against the temporal subsampling account of illusory motion reversal. Journal of Vision, 8(4), 13.1-5. doi:10.1167/8.4.13

Kline, K. A., Holcombe, A. O., \& Eagleman, D. M. (2004). Illusory motion reversal is caused by rivalry, not by perceptual snapshots of the visual field. Vision Research, 44(23), 2653-8. doi:10.1016/j.visres.2004.05.030

Kline, K. A., Holcombe, A. O., \& Eagleman, D. M. (2006). Illusory motion reversal does not imply discrete processing: Reply to Rojas et al. Vision Research, 46(6-7), 1158-1159.

Koch, C. (2004). The Quest for Consciousness: A Neurobiological Approach. Roberts \& Co. 
Lu, Z. L., \& Sperling, G. (1995). Attention-generated apparent motion. Nature, 377(6546), 237239. doi:10.1038/377237a0

Mather, G., Pavan, A., Campana, G., \& Casco, C. (2008). The motion aftereffect reloaded. Trends in cognitive sciences, 12(12), 481-487. doi:10.1016/j.tics.2008.09.002

McComas, A. J., \& Cupido, C. M. (1999). The RULER model. Is this how the somatosensory cortex works? Clinical Neurophysiology, 110(11), 1987-94.

Nishida, S., Sasaki, Y., Murakami, I., Watanabe, T., \& Tootell, R. B. (2003). Neuroimaging of direction-selective mechanisms for second-order motion. Journal of Neurophysiology, 90(5), 3242-3254.

Nishida, S., \& Sato, T. (1995). Motion aftereffect with flickering test patterns reveals higher stages of motion processing. Vision research, 35(4), 477-490.

Purves, D., Paydarfar, J. A., \& Andrews, T. J. (1996). The wagon wheel illusion in movies and reality. Proceedings of the National Academy of Sciences of the United States of America, 93(8), 3693-7.

Rayner, K. (1998). Eye movements in reading and information processing: 20 years of research. Psychological Bulletin, 124(3), 372-422.

Reddy, L., Rémy, F., Vayssière, N., \& VanRullen, R. (2011). Neural correlates of the continuous Wagon Wheel Illusion: a functional MRI study. Human brain mapping, 32(2), 163-170. doi:10.1002/hbm.21007

Rees, G., Frith, C. D., \& Lavie, N. (1997). Modulating Irrelevant Motion Perception by Varying Attentional Load in an Unrelated Task. Science, 278(5343), 1616-1619. doi:10.1126/science.278.5343.1616

Reichardt, W. (1961). Autocorrelation, a principle for the evaluation of sensory information by the central nervous system. In W. A. Rosenblith (Ed.), Sensory Communication (pp. 303-317). Cambridge, MA: MIT Press. 
Rojas, D., Carmona-Fontaine, C., López-Calderón, J., \& Aboitiz, F. (2006). Do discreteness and rivalry coexist in illusory motion reversals? Vision Research, 46(6-7), 1155-1157.

Schouten, J. F. (1967). Subjective stroboscopy and a model of visual movement detectors. In I. Wathen-Dunn (Ed.), Models for the Perception of Speech and Visual Form (pp. 44-55). Cambridge, MA: MIT Press.

Seiffert, A. E., Somers, D. C., Dale, A. M., \& Tootell, R. B. H. (2003). Functional MRI Studies of Human Visual Motion Perception: Texture, Luminance, Attention and After-effects. Cerebral Cortex, 13(4), 340-349. doi:10.1093/cercor/13.4.340

Simpson, W. A., Shahani, U., \& Manahilov, V. (2005). Illusory percepts of moving patterns due to discrete temporal sampling. Neuroscience Letters, 375(1), 23-27.

Snowden, R. J., \& Hess, R. F. (1992). Temporal frequency filters in the human peripheral visual field. Vision Research, 32(1), 61-72.

Vaina, L. M., \& Soloviev, S. (2004). First-order and second-order motion: neurological evidence for neuroanatomically distinct systems. Progress in brain research, 144, 197212. doi:10.1016/S0079-6123(03)14414-7

VanRullen, R. (2006). The continuous Wagon Wheel Illusion is object-based. Vision Research, 46(24), 4091-5. doi:10.1016/j.visres.2006.07.030

VanRullen, R. (2007). The continuous Wagon Wheel Illusion depends on, but is not identical to neuronal adaptation. Vision Research, 47(16), 2143-9. doi:10.1016/j.visres.2007.03.019

VanRullen, R., \& Koch, C. (2003). Is perception discrete or continuous? Trends in Cognitive Sciences, 7(5), 207-213.

VanRullen, R., Pascual-Leone, A., \& Battelli, L. (2008). The continuous Wagon wheel illusion and the 'when' pathway of the right parietal lobe: a repetitive transcranial magnetic stimulation study. PLoS ONE, 3(8), e2911. doi:10.1371/journal.pone.0002911 
VanRullen, R., Reddy, L., \& Koch, C. (2005). Attention-driven discrete sampling of motion perception. Proceedings of the National Academy of Sciences of the United States of America, 102(14), 5291-6. doi:10.1073/pnas.0409172102

VanRullen, R., Reddy, L., \& Koch, C. (2006). The continuous wagon wheel illusion is associated with changes in electroencephalogram power at approximately $13 \mathrm{~Hz}$. The Journal of Neuroscience, 26(2), 502-7. doi:10.1523/JNEUROSCI.4654-05.2006 